\title{
Hipercalcemia Tumoral. A Propósito de Tres Casos
}

\author{
KATHERINE KOPP G. ${ }^{1}$, FERNANDO BRACHO M. ${ }^{2}$, EMMA CONCHA V. ${ }^{1}$, \\ NIMIA VALLEJOS C. ${ }^{1}$, CAROLINA NICKLAS D. ${ }^{2}$, MILENA VILLARROEL C. ${ }^{1}$ \\ 1. Hemato-Oncóloga infantil, Unidad de Oncología Infantil Hospital Luis Calvo Mackenna. Santiago de Chile. \\ 2. Becado de sub-especialidad en Hematología y Oncología Infantil. Departamento de Pediatría y Cirugía Infantil. \\ Sede oriente. Facultad de Medicina Universidad de Chile.
}

\begin{abstract}
Tumor Related Hypercalcemia: A Review of Three Cases

Hypercalcemia is an infrecuent complication in pediatric oncology, with an incidence between 0,5 and $3 \%$. It can occur at diagnosis, during the disease course or at relapse, and it is resolved by treating the underlying pathology, requiring in some cases the use of specific therapy such as calcitonin and biphosphonates. This article presents 3 cases of children with cancer and hypercalcemia during their illness, analyzing its clinical presentation, pathophysiology and treatment.

(Key words: Tumoral hypercalcemia, pediatric oncology, paraneoplasic syndrome).

Rev Chil Pediatr 2010; 81 (4): 347-352
\end{abstract}

\section{RESUMEN}

La hipercalcemia es una complicación infrecuente en niños con cáncer, con una incidencia que oscila entre 0,5 y $3 \%$. Se puede presentar al diagnóstico, durante el tratamiento o en la recaída de una neoplasia, se resuelve al tratar la enfermedad de base, pudiendo además, requerir el uso de terapia específica como calcitonina y bifosfonatos. En el presente artículo se revisan 3 casos clínicos de niños con cáncer que presentaron hipercalcemia en algún momento de su enfermedad, se discute su forma de presentación, fisiopatología y manejo.

(Palabras clave: Hipercalcemia Tumoral, cáncer Infantil, Síndrome paraneoplásico).

Rev Chil Pediatr 2010; 81 (4): 347-352

\section{Introducción}

En las neoplasias, las alteraciones metabólicas agudas pueden determinar complicaciones de riesgo vital y entre ellas, la hiper- calcemia es la complicación más frecuente, se presenta como síndrome paraneoplásico hasta en un $30 \%$ de los casos de adultos con cáncer ${ }^{1}$. En pediatría la hipercalcemia asociada a cáncer es rara, se describe en menos del 3\% de

Trabajo recibido el 13 de noviembre de 2009, devuelto para corregir el 28 de diciembre de 2009, segunda versión el 24 de mayo de 2010, aceptado para publicación el 01 de junio de 2010.

Correspondencia a:

Katherine Kopp G.

E-mail: katitakopp@yahoo.com 
los pacientes $^{2}$, a pesar de su baja frecuencia, su hallazgo en un niño previamente sano debe hacer considerar la presencia de una neoplasia subyacente. Se ha descrito su presencia en asociación con leucemias y linfomas, así como también en neoplasias con metástasis óseas, siendo más rara de ver en tumores óseos pri$\operatorname{marios}^{2,3}$.

La hipercalcemia asociada a cáncer se resuelve con el tratamiento específico de la neoplasia subyacente, sin embargo, esta puede llegar a ser severa y constituir una complicación grave con riesgo vital, por lo que muchas veces se requieren medidas adicionales para disminuir en forma rápida los niveles de calcio sanguíneo ${ }^{3}$.

El presente reporte, tiene por objetivo estudiar la hipercalcemia como forma de presentación de una neoplasia en niños. Se realiza una revisión de su fisiopatología, forma de presentación y manejo.

\section{Material y Método}

Se presentan, 3 casos clínicos de niños con hipercalcemia asociada a cáncer, dos de ellos al diagnóstico y uno durante el tratamiento, evidenciando una recaída.

\section{Caso 1}

Paciente de 12 años, sexo femenino, sin antecedentes mórbidos. Historia de un mes de evolución con dolor abdominal, vómitos y baja de peso $(3 \mathrm{~kg})$. Consulta en el Servicio de Urgencia por vómitos y deshidratación; los exámenes iniciales: calcio total $16,4 \mathrm{mg} / \mathrm{dl}$, calcio iónico $2,0 \mathrm{mmol} / \mathrm{L}$, fósforo $3,6 \mathrm{mg} / \mathrm{dl}$, creatinina $1,62 \mathrm{mg} / \mathrm{dl}$, ácido úrico $12,5 \mathrm{mg} / \mathrm{dl}$, hormona paratiroídea (PTH, intacta, Laboratorio Barnaffi, equipo Imulete 2000 de Siemens, $\mathrm{VN}$ adultos 12-15 pg/ml, Quimioluminicencia directa) $11,8 \mathrm{pg} / \mathrm{ml}$ (normal), fórmula hematológica con 15000 glóbulos blancos, sin anemia o trombopenia. Se plantea insuficiencia renal por deshidratación y se maneja en Unidad de Cuidado Intensivo Pediátrico (UCIP) con hiperhidratación, furosemida e hidrocortisona intravenosa, luego de 4 días se logra normali- zar la calcemia. En un segundo hemograma se describe $2 \%$ de blastos, por lo que se sospecha neoplasia hematológica; el mielograma evidencia infiltración leucémica por $30 \%$ de linfoblastos FAB-L1; Inmunofenotipo: Leucemia Linfoblástica Aguda estirpe B, común.

\section{Caso 2}

Paciente de 14 años, sexo masculino, con antecedentes de déficit atencional e hipotiroidismo. Historia de un mes de evolución con compromiso del estado general, artralgias, inapetencia y baja de peso $(7 \mathrm{~kg})$. Los exámenes iniciales: calcio total $17,3 \mathrm{mg} / \mathrm{dl}$, calcio iónico $1,83 \mathrm{mmol} / \mathrm{L}$, fósforo: $3,4 \mathrm{mg} / \mathrm{dl}$, creatinina $1,57 \mathrm{mg} / \mathrm{dl}$, ácido úrico $12,2 \mathrm{mg} / \mathrm{dl}, \mathrm{LDH}$ 5947 U/L, hemograma con 25200 glóbulos blancos, 22\% de blastos; mielograma: infiltración leucémica, 95\% de linfoblastos FABL1; Inmunofenotipo: Leucemia Linfoblástica Aguda pre-B. Requiere manejo en UCIP con hiperhidratación, furosemida, calcitonina y bifosfonatos (pamidronato) para lograr reducir la calcemia. Recibe prednisona como tratamiento de su leucemia y desde la segunda semana de terapia, evoluciona con hipocalcemia e hipofosfemia que requiere reposición oral por un mes.

\section{Caso 3}

Paciente de 5 años, portador de Méduloblastoma de fosa posterior con metástasis espinales al diagnóstico; tratado con cirugía, radioterapia y quimioterapia; con remisión de la enfermedad. Luego del séptimo ciclo de quimioterapia, presenta dolor en la rodilla derecha, la radiografía evidencia lesión lítica en el tercio proximal de la tibia; exámenes: calcio total $16 \mathrm{mg} / \mathrm{dl}$, calcio iónico $1,9 \mathrm{mmol} / \mathrm{L}$, fósforo $4,2 \mathrm{mg} / \mathrm{dl}$, creatinina $0,6 \mathrm{mg} / \mathrm{dl}$, ácido úrico $8 \mathrm{mg} / \mathrm{dl}$, hemograma con trombocitopenia, por lo que se realiza mielograma que demuestra infiltración tumoral de médula ósea. La resonancia magnética de cerebro y columna espinal con recaída de la enfermedad de base. Se logra controlar la hipercalcemia con hidratación, furosemida y prednisona $(2 \mathrm{mg} / \mathrm{kg} /$ día $)$; se inicia quimioterapia para su recaída tumoral sin lograr remisión. 


\section{Discusión}

Estos 3 casos clínicos, ilustran como el cáncer en los niños puede presentarse con hipercalcemia, una vez reconocida la neoplasia subyacente, no fue necesaria la medición de PTH, PTHrP u otros exámenes relacionados con el metabolismo del calcio.

El tratamiento de soporte general y terapia oncológica específica lograron controlar la hipercalcemia en estos pacientes.

La hipercalcemia es la elevación del calcio sérico por sobre $10,5 \mathrm{mg} / \mathrm{dl}$. Se puede originar por movilización excesiva desde el tejido óseo (depósito), por aumento de la absorción en el tracto digestivo, disminución en su excreción renal o la combinación de estos mecanismos ${ }^{4}$.

Las manifestaciones clínicas de una hipercalcemia son variadas, a nivel gastrointestinal puede causar dolor abdominal, constipación, anorexia, náuseas y vómitos; a nivel cardiovascular, se produce aumento de la contractibilidad cardíaca y alteraciones electrocardiográficas, como prolongación del intervalo P-R y acortamiento del Q-T; las manifestaciones musculares incluyen hipotonía e hiporreflexia; el compromiso del sistema nervioso central puede ir desde cambios de personalidad hasta el estupor y coma; a nivel renal se produce un daño tubular reversible, lo que condiciona una incapacidad para concentrar la orina y poliuria $^{4}$.

En general, el diagnóstico diferencial de la hipercalcemia se centra en diferenciar si se trata de un hiperparatiroidismo primario o de una neoplasia, excluyendo otras causas extremadamente raras como la intoxicación por vitamina D. Para esto es útil la determinación de $\mathrm{PTH}$, la que se encontrará elevada en un hiperparatiroidismo y normal en una hipercalcemia asociada a una neoplasia. Por otro lado, los niveles plasmáticos elevados del péptido relacionado con la hormona paratiroidea (PTHrP), acompañado de hipofosfemia son propios de una hipercalcemia tumoral ${ }^{1}$.

\section{Fisiopatología de la hipercalcemia asociada al cáncer}

El control del calcio sérico normal $(8,9-$ $10,3 \mathrm{mg} / \mathrm{dl}$ ) depende de 3 sistemas hormonales que determinan el intercambio de calcio entre intestino, riñón, hueso y líquido extracelular. Ellos son la PTH, 1,25 (OH)-vitamina D y calcitonina. Cada uno de ellos controla directa o indirectamente el calcio sérico mediante mecanismos de retroalimentación ${ }^{5}$.

La PTH impide la caída del calcio sérico estimulando su reabsorción a nivel renal, aumentando la resorción ósea y estimulando la producción de $1,25(\mathrm{OH})$-vitamina $\mathrm{D}$ en el riñón, lo que aumenta la absorción de calcio a nivel intestinal. La calcitonina inhibe la resorción ósea, con lo cual actúa como un contra regulador de la $\mathrm{PTH}^{4,5}$.

La mayoría del calcio en el cuerpo humano se encuentra en forma de depósitos inorgánicos en el hueso, los que pueden ser movilizados a la circulación sistémica mediante un proceso llamado resorción ósea ${ }^{6}$.

La resorción ósea (figura 1), es llevada a cabo por los osteoclastos, células derivadas de un precursor del sistema monocítico-macrofágico que se diferencia a un osteoclasto inactivo. La actividad de los osteoclastos está regulada por factores locales y sistémicos, entre los sistémicos tenemos PTH, 1,25 (OH)vitamina $\mathrm{D}$ y tiroxina; a nivel local, participan el microambiente óseo, IL-6 y prostaglandinas producidas por los osteoblastos, además de algunas citoquinas activadoras e inhibidoras sintetizadas por linfocitos T. Todos estos factores estimulan en la célula estromal del hueso la expresión del ligando de factor activador nuclear (RANKL), el que se uniría al receptor de RANK presente en el osteoclasto inactivo, activándose a través de una señal nuclear de tipo tirosin-kinasa (NFkB). El resultado final es un osteoclasto activo que realiza el proceso de resorción ósea ${ }^{7}$.

La hipercalcemia tumoral (figura 2) se produce fundamentalmente por una excesiva resorción ósea, la que en la mayoría de los casos, depende de factores humorales que la estimulan. Entre ellos, el más importante, es el PTHrP, seguido por la sobreproducción a nivel local de algunas citoquinas. La PTH raramente se secreta en forma ectópica y generalmente no está involucrada en la génesis de este tipo de hipercalcemia ${ }^{8}$.

Normalmente, el PTHrP actúa como un 

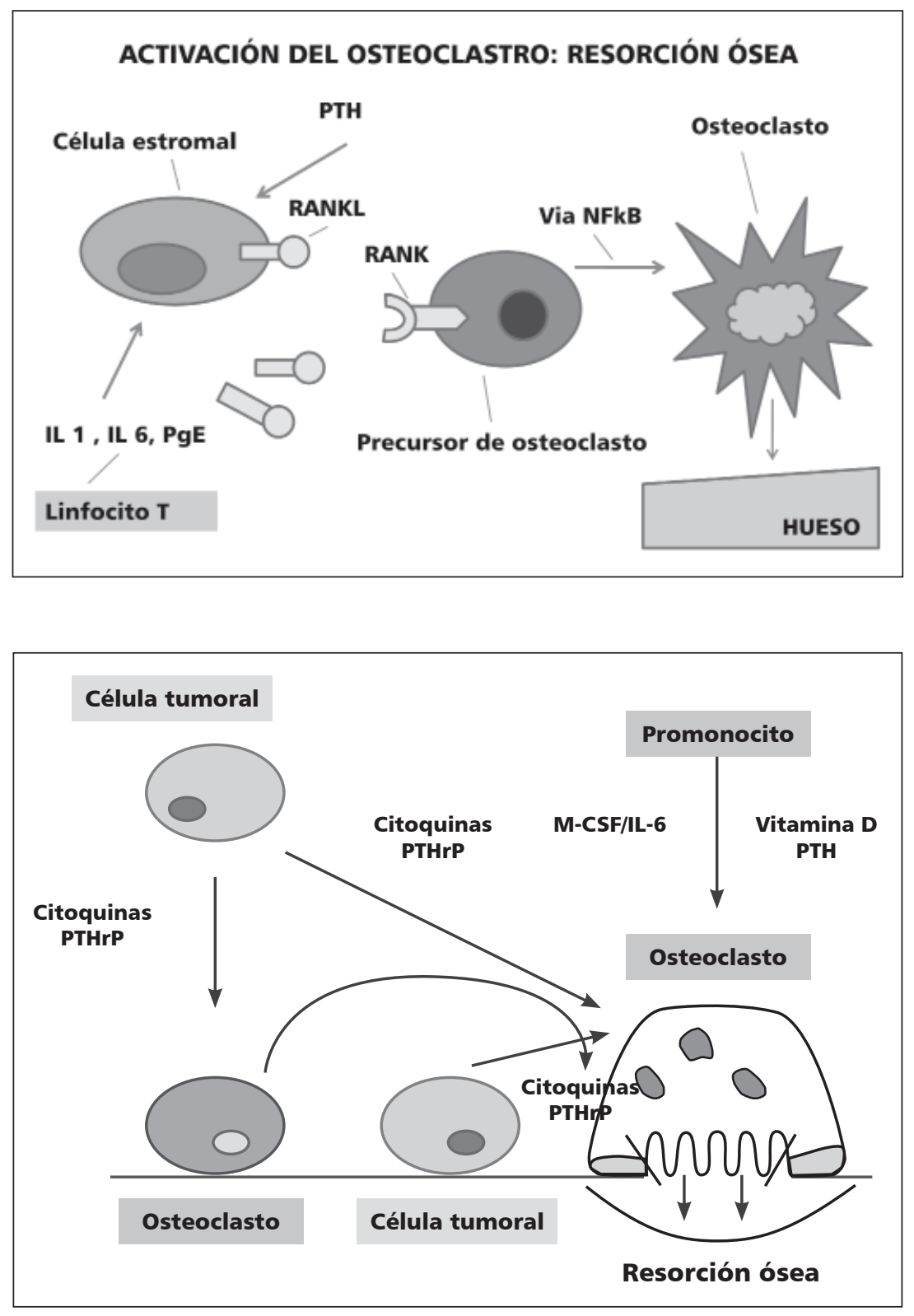

Figura 1. Fisiología de la resorción ósea. factor tisular que regula la proliferación y diferenciación celular en el desarrollo fetal y su estructura es muy parecida a la PTH, por lo que puede unirse a su receptor y así remedar sus funciones ${ }^{1}$. Cuando un tumor secreta PTHrP, esta puede actuar en el receptor de PTH del hueso, estimulando la resorción ósea y movilizando calcio. A nivel renal el PTHrP produce un aumento de la reabsorción renal de calcio y un aumento de la eliminación de fosforo ${ }^{7}$.
El aumento de resorción ósea puede ocurrir también por la acción a nivel local de factores sistémicos como citoquinas u otros mediadores producidos por células tumorales ubicadas a distancia (mecanismo endocrino) o por la producción local (en el hueso) de estos mediadores secundaria a una infiltración tumoral (mecanismo paracrino). De todas formas, no todas las metástasis o infiltraciones tumorales óseas producen hipercalcemia, por lo que se 
postula que es necesaria la producción local o sistémica de ciertos mediadores como IL-1, TNF-alfa, linfrotoxina, PGE2 o IL-6 $6^{7,9}$.

En relación a la hipercalcemia observada en leucemias, en algunos casos el mecanismo sería la producción anómala de PTHrP y en otros la producción aberrante de citoquinas como IL-1, IL-6 y TNF- $\alpha^{9,10}$. Se han descrito casos de LLA pro- $\mathrm{B}$, con $\mathrm{t}(17 ; 19)$, en las que se produce una proteína quimérica (E2A-HLF) que estimularía la sobreproducción de de Anexina II e IL-1, esta última asociada a hipercacemia tumoral ${ }^{11}$. En el caso de los tumores sólidos, con o sin metástasis óseas, se puede producir hipercalcemia mediante la producción de PTHrP o citokinas hipercalcemiantes ${ }^{7}$.

\section{Tratamiento}

La mejor forma de corregir una hipercalcemia tumoral es tratar la neoplasia subyacente, pero los pacientes con hipercalcemia severa y sintomática, requieren muchas veces de un tratamiento destinado a reducir en forma rápida los niveles de calcio sanguíneo, dado que el tratamiento oncológico específico requiere de algunos días para revertir la hipercalcemia ${ }^{12}$.

Dependiendo de la severidad de la hipercalcemia y sus síntomas, se requiere instaurar medidas para reducir los niveles de calcio sanguíneo, las que van, desde la hidratación y uso de diuréticos para los casos leves, hasta el manejo en UCIP y hemodiálisis, para los casos severos.

Entre las medidas y fármacos efectivos en la reducción de la calcemia tenemos:

1) Hiperhidratación: Volumen de $3000-4000$ $\mathrm{cc} / \mathrm{m}^{2} /$ día, lo que evita la deshidratación y permite una diuresis adecuada.

2) Diuréticos de asa: Furosemida, permite aumentar la excreción urinaria de calcio.

3) Calcitonina: Actúa sobre el hueso inhibiendo la resorción ósea y a nivel renal estimula la eliminación urinaria de calcio. Tiene un efecto rápido (en pocas horas), se utiliza en dosis de 4 a 8 U/kg/día, IM o SC, en 2 ó 3 dosis. Su efecto hipocalcemiante dura 2 a 3 días, por lo que generalmente se asocia a otros fármacos ${ }^{12}$.
4) Corticoides: Son muy efectivos en reducir la calcemia, sin embargo, se requiere haber descartado una neoplasia hematológica (ej. Leucemia), dado que su uso puede entorpecer su diagnóstico. Es importante tener en cuenta que existe el riesgo de producir un síndrome de lisis tumoral si no se usa con precaución.

5) Bifosfonatos (pamidronato): son efectivos en la reducción de una hipercalcemia severa, pero de efecto lento ( 24 a $48 \mathrm{hrs)} \mathrm{y} \mathrm{muy}$ prolongado (semanas), lo que puede provocar en forma paradojal una hipocalcemia una vez controlada la neoplasia subyacente $^{12}$.

6) Hemodiálisis: En casos de hipercalcemia extrema y refractaria a otras medidas.

Cuando se trata de una hipercalcemia leve, es suficiente la hidratación y el uso de diuréticos para controlar el cuadro, sin embargo, cuando la hipercalcemia es severa, se requiere combinar algunas de estas medidas, en espera de que el tratamiento oncológico específico logre controlar el cuadro de base.

En suma, si bien la hipercalcemia asociada a una neoplasia en niños es poco frecuente, cuando aparece, puede poner en riesgo la vida del paciente y su asociación con un cáncer; requiere un alto grado de sospecha clínica de parte del pediatra general; sólo de este modo se logrará un diagnóstico precoz y adecuado de un cáncer, esencial para un tratamiento exitoso.

\section{Referencias}

1.- Forga L, Anda E, Martinez JP: Síndromes hormonales Paraneoplásicos. An Sist Sanit Navarra 2005; 2: 213-26.

2.- Kerdulo C, Aerts I, Fattet $S$, et al: Hypercalcemia and Childhood Cancer. J Pediatr Hematol Oncol 2005; 27: 23-7.

3.- Mathur M, Sykes JA, Saxena VR, Rao SP, Goldman GM: Treatment of acute lymphoblastic leukemia-induce extreme hypercalcemia whith pamidronato and calcitonin. Pediatr Crit Care Med 2003; 4: 252-5.

4.- Rodríguez P: Diagnóstico y tratamiento de la hipercalcemia En: Endocrinología clínica, Santiago: Editorial Mediterránea 2000; cap 32: 188-91. 
5.- Rodríguez P: Metabolismo del calcio, fósforo, magnesio y hormonas calciotrópicas. En: Endocrinología clínica, Santiago: Editorial Mediterránea 2000; cap 31: 183-7.

6.- Cohen M: The new bone biology. Am J Med Genetic 2006; 140A: 2646- 706 .

7.- Roodman D: Mechanisms of bone metastasis. N Engl J Med 2004; 350: 1655-64.

8.- Lankisch P, Kramm C, Hermsen D, Wessalowski R: Hypercalcemia whit nephrocalcinosis and impaired renal function due to increased parathyroid hormone secretion at onset of childhood acute lymphoblastic leukemia. Leukemia\& Lymphoma 2004; 45 (8): 1695-7.

9.- Niizuma H, Fujii K, Sato A, Fujiwara I, Tekeyama J, Imaizumi M: PTHrP-independent hypercalcemia whit increased proinflammatory cytokines and bone resorption in two children whit CD19 negative precursor B
Acute Lymphoblastic Leukemia. Pediatr Blood Cancer. 2006; 49 (7): 990-3.

10.- Shimonodan H, Nagayama J, Nagatoshi Y, Hatanaka M, Tokada A, Iguchi H: Acute Lymphoblastic Leukemia in Adolescence whit multiple osteolytic lesión and hypercalcemia mediated by lymphoblast-producing parathyroid hormone - related peptide: a case report and review of the literature. Pediatr Blood Cancer 2005; 45: 333-9.

11.- Matsunaga T, Inaba T, Matsui H, Okuya M, Miyajima A, Inukai T: Regulation of annexin II by cytokine - initiated signaling pathways and E2A - HLF oncoprotein. Blood 2004; 103 (8): 3185-90.

12.- Smidt I, Stachel D, Schön C, Bouer M, Haas RJ: Pamidronate and calcitonin as therapy of acute cancer related hypercalcemia in children. Klin Pädiatr 2001; 213: 30-4. 